\title{
La implantación neoliberal de la sojización transgénica en Argentina. Paquete tecnológico, patentes y consecuencias indeseables previsibles'
}

Universidad Nacional de General Sarmiento Universidad de Buenos Aires

Artículo de Reflexión derivado de Investigación

Recibido: agosto 21 de 2015 - Aprobado: octubre 21 de 2015

\section{Resumen}

Recorreremos algunos aspectos de la implantación de la soja transgénica en Argentina y las consecuencias indeseables previsibles que derivaron por aprobarse el uso del paquete tecnológico sin haber realizado estudios interdisciplinarios de impacto ambiental y social. Esa implantación fue posibilitada en el contexto del dispositivo de poder neoliberal, mercados autorregulados y una nueva división internacional del trabajo que buscó explícitamente que Argentina reprimarizara su matriz productiva y siviera como plataforma productiva para multinacionales alimenticias. Para este

I El presente artículo deriva de una sublínea del proyecto de investigación "La democracia constitucional: entre la corrupción global y el poder comunicativo" financiado por la Universidad Nacional de General Sarmiento (30/3/33), llevado a cabo entre 20 I I y 2014 con la dirección de quien suscribe, investigador y profesor de la mencionada universidad. En el contexto del proyecto mencionado nos ocupamos del conflicto entre el derecho al conocimiento y el derecho a la propiedad intelectual en el modo de patentes de invención, así como a la opacidad institucional al autorizarse la soja RR en Argentina.

2 Profesor por concurso público, titular de Filosofía del Derecho (UNGS). Profesor por concurso público, titular de Derecho y Economía de los Medios. Profesor por concurso público, asociado a cargo de una cátedra de Introducción al Pensamiento Científico (UBA). Dirección electrónica: flax@ungs.edu.ar 
propósito, el Acuerdo sobre ADPIC juega un papel fundamental para controlar el mercado de alimentos a través de las patentes de los OGM.

Palabras clave: Biotecnologías, OGM, patentes, neoliberalismo, mercados autorregulados, división internacional del trabajo, brecha tecnológica, sojización, interdisciplina.

\title{
The neo-liberal implantation of transgenic soy-ization in Argentina. Technological package, patents and foreseeable undesirable consequences
}

\begin{abstract}
We will journey along some aspects of the implantation of transgenic soy in Argentina and the foreseeable undesirable consequences that were brought about by having approved the use of the technological package without first having carried out interdisciplinary studies of social and environmental impact. This implantation was made possible within the context of the neo-liberal power display, self-regulated markets and a new international work division that sought that Argentina re-primarized its productive matrix and served as a productive platform for food multi-national companies. For this purpose, the Agreement about DDPIC plays an important role to control the food market through the patents of OGM.
\end{abstract}

Key words: bio-technologies, OGM, patents, Neo-liberalism, international work division, technological gap, soy-ization, inter-discipline.

\section{A implantação neoliberal da sojização transgênica na Argentina. Pacote tecnológico, patentes e consequências indesejáveis previsíveis}

\begin{abstract}
Resumo
Vamos percorrer alguns aspectos da implantação da soja transgênica na Argentina e as consequências indesejáveis previsíveis que derivaram por aprovar o uso do pacote tecnológico sem ter realizado estudos interdisciplinares de impacto ambiental e social. Essa implantação foi possibilitada no contexto do dispositivo de poder neoliberal, mercados autorregulados e uma nova divisão internacional do trabalho que procurou explicitamente que Argentina re- primarizara sua matriz produtiva e servira como plataforma produtiva para multinacionais alimentares. Para este propósito, o Acuerdo sobre ADPIC joga um papel fundamental para controlar o mercado de alimentos a través das patentes dos OMG.
\end{abstract}

Palavras chave: biotecnologias, OGM, patentes, neoliberalismo, mercados autorregulados, divisão internacional do trabalho, brecha tecnológica, sojização, interdisciplina. 


\section{El conocimiento como mercancía: el Acuerdo sobre ADPIC}

La ingeniería genética y las biotecnologías derivadas, incluida la transgénesis, abrieron enormes posibilidades para mejorar la calidad de vida de la población del planeta, tanto en lo que tiene que ver con la expectativa de cura de enfermedades, como en lo que a la producción de alimentos se refiere. Por lo cual, resultaría retrógrado e irresponsable oponerse a las mismas en bloque. Sin embargo, estas posibilidades pueden frustrarse si las biotecnologías se ponen meramente al servicio del mercado. Peor aún, si se implementan sin una evaluación reflexiva, crítica e interdisciplina de las consecuencias indeseables que puede generar en algunos casos la incorporación de OGM (organismos genéticamente modificados), como veremos en un caso real de gran escala, a saber, la sojización en Argentina.

Asimismo, no puede desatenderse que las biotecnologías se desarrollan en un marco jurídico en el cual las patentes de invención convierten al conocimiento científico y tecnológico en una mercancía más. Más aún, se aplican en el contexto de un dispositivo de poder neoliberal que conduce a una concentración cada vez mayor de la riqueza, dejando afuera del disfrute de los avances científicos y tecnológicos a vastos sectores de la población mundial. Este dispositivo de poder tiene componentes económicos, políticos, mediáticos e intelectuales que sostienen una idea de libertad como mera ausencia de interferencias, con el propósito de instalar la concepción de los mercados autorregulados, es decir, no regulados por los Estados. ${ }^{3}$ Esto facilita que en aquellos países en los que el Estado renunció a regular algunas materias o es sensible a las presiones externas, sea sumamente fácil implementar iniciativas motivadas meramente por las ganancias rápidas, sin evaluarse eventuales consecuencias perjudiciales. Por otro lado, la concepción que defiende la ausencia de regulaciones es falaz, dado que las regulaciones estatales se reemplazan por reglas de juego que constituyen re-regulaciones o meta regulaciones a la medida de los grandes jugadores, las cuales son establecidas por organismos internacionales como la Organización Mundial de Comercio (OMC), por iniciativa de países y corporaciones multinacionales que tienen ventajas comparativas en cuestiones científicas, tecnológicas y financieras. Tal es el caso por ejemplo del Acuerdo sobre Aspectos de la Propiedad Intelectual Vinculados con el Comercio (ADPIC), establecido en 1994, en plena hegemonía neoliberal. El mencionado tratado pretendió -y logró en buena medida- que prácticamente todo el conocimiento y la cultura fueran convertidos en mercancía y subsumidos bajo sus reglas. Esto pudo revertirse en buena medida con respecto a las expresiones culturales, a través de la Convención para la protección y promoción de la diversidad de las expresiones culturales, en 2005, pero sigue vigente con pocas modificaciones con respecto al conocimiento científico aplicado y a las tecnologías.

El derecho a la propiedad es un derecho fundamental, pero también lo es el derecho al conocimiento y el derecho a la satisfacción de las necesidades básicas. Entre los derechos fundamentales pueden existir conflictos, denominados "casos difíciles" o "insólitos" que requieren armonizar o

3 Al respecto nos referimos en Flax, Javier Ética, política y mercado. En torno a las ficciones neoliberales, Los Polvorines, UNGS, 20 I3, con reedición en 20I4. Está disponible su versión digital en http:/www.unesco.org.uy/shs/red-bioetica/es/biblioteca/libros.html 
establecer prioridades entre los mismos. ${ }^{4}$ Entre estos conflictos entre derechos fundamentales uno sumamente relevante por su incidencia en el desarrollo científico, tecnológico y productivo de los países, es el conflicto entre el derecho al conocimiento y el derecho a la propiedad intelectual bajo la forma de patentes de invención, dado que éstas últimas generan serios obstáculos para el avance del conocimiento por las barreras que generan los monopolios artificiales. Dirimir, transformar o buscar un equilibrio en este conflicto normativo nos lleva al campo reflexivo de la ética y al campo de la política. A nuestro juicio, se requiere distinguir aquellos conocimientos que tienen por objeto la satisfacción de necesidades básicas, de aquellos conocimientos orientados a los bienes suntuarios. ${ }^{5}$ En el primer caso hay que priorizar el derecho a los frutos del conocimiento por sobre el derecho a la propiedad intelectual.

Por ello, la incorporación de algunas biotecnologías debe considerarse en un marco amplio que considere en cada caso sus enormes ventajas, pero también que evalúe muchas de las consecuencias indeseables previsibles, a través de un enfoque interdisciplinario e, incluso, un debate público que excede las competencias de los biotecnólogos. Podemos pensar hipotéticamente que se produce un OGM incuestionablemente benéfico. Pero al patentarse y generarse un monopolio sobre ese OGM las consecuencias podrían ser sumamente perjudiciales en términos de bien público y de calidad de vida. Por eso, dejar en manos de los expertos biotecnólogos la política pública en torno a la aplicación de las biotecnologías es equivalente a dejar la política económica exclusivamente en manos de economistas o la política de defensa en manos exclusivamente de militares. Más aún, dejar la política pública en manos de expertos de cada especialidad no es lo que corresponde en un Estado constitucional de derecho, atendiendo a que existen objetivos valiosos y metas disvaliosas cuyo conocimiento específico no se puede determinar desde la experticia científica ni tecnológica. ${ }^{6}$ Qué es valioso y qué es disvalioso es originalmente un problema de la ética, pero luego se plasma en términos de derechos fundamentales en los pactos internacionales, obligantes para la mayoría de las naciones. Algunos de esos pactos internacionales, a su vez, pasan a constituir el fundamento sustantivo del derecho positivo en algunos países, como es el caso de Argentina, que los incorpora como normas constitucionales. En consecuencia, tratar las posibilidades de las biotecnologías y sus aplicaciones constituyen problemas éticos y políticos y no, meramente, problemas biotecnológicos. Por su parte, no atender los condicionamientos que generan las patentes, puede conducir a enfoques erróneos. A su vez, se requiere comprender el papel que juegan las patentes en el contexto del

$4 \quad$ Al respecto puede verse Alexy, Robert El concepto y la validez del derecho, Barcelona, Gedisa, I991.

5 Puede verse en Flax, Javier. Política científica, interdisciplina y derechos humanos, Buenos Aires, Biblos, 20I4, reeditado en 20 I5. Capítulo 3 "Patentes: un conflicto entre el derecho a la propiedad y el derecho al conocimiento".

6 Efectivamente, la racionalidad científica es o bien deductiva o bien inductiva o bien instrumental. La racionalidad científica, en tanto deductiva no puede fundamentar principios valorativos porque estos constituyen la premisa mayor. Inductivamente no se pueden fundamentar los valores sin incurrir en una falacia naturalista que consiste en considerar válido lo que es meramente vigente. Finalmente, la racionalidad técnica o instrumental sólo puede evaluar medios para alcanzar un objetivo valioso, pero éste la antecede y queda fuera de su campo. En consecuencia, la fundamentación de objetivos valiosos corresponde a otro campo de saber: la moral y la ética, como reflexión sobre la moral, las cuales recurren a otros tipos de racionalidad, comunicativa, abductiva, según el caso. 
actual dispositivo de poder-saber neoliberal, en la medida en que las reglas de juego vigentes fueron establecidas atendiendo al reforzamiento de las ventajas competitivas de aquellos países y corporaciones con capacidad, no sólo para generar los conocimientos, sino también para patentarlos, lo cual no es idéntico, debido al financiamiento adicional y al apoyo jurídico necesarios. ${ }^{7}$ Desde nuestro punto de vista, aquellos enfoques que pretenden poder dilucidar estas cuestiones dentro del marco de la propia disciplina incurren en una visión reductiva de la cuestión. En otros casos pueden existir visiones ingenuas, visiones cientificistas y, lamentablemente, también existen visiones interesadas en que estas cuestiones no se traten fuera de los ámbitos opacos en los que se toman las decisiones. ${ }^{8}$

Atendiendo algunas de las posibles y efectivas aplicaciones de las biotecnologías, debemos atender la incidencia que pueden tener las mismas en la producción de alimentos. Pero también, el modo en que las patentes aumentan la brecha tecnológica y la dependencia entre los países. Si bien el conocimiento científico y tecnológico es más accesible para los países en vías de desarrollo, las patentes de invención generan barreras artificiales para la innovación que juegan en contra de esos países a los que se le vende ese conocimiento a valores abusivos, propios de posiciones de dominio en el mercado o de monopolios sobre el conocimiento, generados por las patentes. ${ }^{9}$

$7 \quad$ El patentamiento es sumamente complicado porque se requiere saber si existió o no un patentamiento previo o si se está patentando lo mismo simultáneamente. Los registros de patentamientos están incompletos y sólo en USA hay 120.000 patentamientos anuales. Asimismo, una patente internacional es muy costosa, por lo que el investigador por sí mismo difícilmente puede hacerlo. Así es que suele quedarse con los derechos quien financia y no el investigador. Se generan muchas controversias y quienes cuentan con mejores estudios de abogados tienen ventajas al momento de dirimirlas, lo cual no deja de ser también una cuestión de financiamiento. Con las reglas actuales, se debe patentar, eventualmente para luego liberar el conocimiento a través de Creative Commons u otro sistema o, como hicieron muchos investigadores, donar las patentes a entidades de bien público.

8 El matemático y filósofo político de la ciencia argentino, Oscar Varsavsky, en su clásico libro Ciencia, política y cientificismo, Buenos Aires, CEAL, 1969, (reeditado en Varsavsky, Obras escogidas, publicada por Ediciones de la UNLa, Lanús, 20I2). denominó "cientificistas" a aquellos científicos que no reflexionan sobre su propia práctica de investigación y no se hacen responsables por las consecuencias indeseables de sus investigaciones. En estas cuestiones pudimos observar diferentes tipos de cientificistas. En un extremo, biotecnólogos intelectualmente honestos, enamorados de las posibilidades de experimentación y creación que les brinda su conocimiento, enamoramiento que se convierte en un obstáculo para reflexionar y comprender sus implicancias. En otro extremo, observamos a aquellos con responsabilidades en políticas públicas y, a la vez, con intereses en empresas biotecnológicas. En estos casos, se trata de una modalidad sofisticada de la denominada "puerta giratoria" que consiste en ocupar cargos públicos para defender intereses particulares.

9 Joseph Stiglitz, economista indudablemente liberal y Premio Nobel de Economía expone de un modo sumamente pormenorizado y esclarecedor que las patentes de invención, lejos de favorecer la innovación científica y tecnológica, constituyen barreras para la investigación a través de la generación de monopolios sobre los conocimientos patentados. De ese modo se desalienta la investigación, pues en la lógica competitiva que establecen esas reglas de juego, el que llega primero se queda con todo. Por ello defiende la arquitectura abierta y cooperativa del conocimiento científico. Además, porque los conocimientos patentados se basan en conocimientos científicos básicos no patentables, financiados generalmente por el erario público a nivel mundial. Al respecto puede verse Stiglitz, Joseph Cómo hacer que funcione la globalización, Buenos Aires, Taurus, 2006, Capítulo "Patentes, beneficios y personas". 


\section{La aprobación opaca de la sojización transgénica}

\section{El agro business y el retorno del modelo agro exportador}

En el caso de las semillas transgénicas, la incorporación de las mismas generó enormes ventajas económicas en términos de rentabilidad para diferentes eslabones de la cadena productiva y comercial, pero a la vez produjo una cantidad de consecuencias indeseables previsibles que, deliberadamente, no se ponderaron ni se consideraron. Así como la implantación de semillas bajo derechos de propiedad intelectual en algunos países se llevó a cabo a partir de imposiciones de denominados Acuerdos de Libre Comercio bilaterales, el cambio en la matriz agropecuaria de un país como la Argentina, se realizó sin someter las transformaciones al escrutinio público ni a las instancias legislativas de gobierno y sin realizarse estudios interdisciplinarios de impacto ambiental, económico y social. Esto ocurrió poco tiempo después del reforzamiento de la patentes de invención a partir del denominado Acuerdo sobre ADPIC (aspectos de la propiedad intelectual vinculados con el comercio) en 1994 en el marco de la OMC, en plena hegemonía neoliberal. Efectivamente, en 1996 la Secretaría de Agricultura, Ganadería y Pesca aprobó las resoluciones que posibilitaron el uso de la soja RRI, es decir, del núcleo del paquete tecnológico que posibilitaría el aumento exponencial de la sojización transgénica. De ese modo, Argentina fue uno de los primeros países en aprobar e incorporar masivamente los transgénicos a la explotación agraria.

Atendiendo a las ventajas competitivas del agro argentino, algunos sectores se expresaron explícitamente a favor de una reprimarización de la economía argentina, de modo de volver a un modelo básicamente agroexportador, dentro del cual se consideraba a la soja en un país como la Argentina, equivalente al petróleo en un país como Kuwait. Desde fines de los años '80 se venía promoviendo la idea de volver a la Argentina agroexportadora. El ex Presidente del Banco Central durante la dictadura genocida y futuro superministro de economía, Domingo Cavallo, por entonces miembro de la Fundación Mediterránea, propuso durante el gobierno de Raúl Alfonsín "volver al modelo agroexportador que tuvo la Argentina en el pasado". Finalmente, se logró instalar en la década del '90, cuando -luego de la dictadura- el neoliberalismo se reinstaló en el gobierno de Argentina.

Inmediatamente aprobado el Acuerdo sobre ADPIC se comenzó a trabajar en la aprobación del uso de la soja RRI (resistente al Roundup, marca comercial del glifosato), para lo cual se instaló un fuerte lobby, incluso a través del aparato publicitario de los multimedia ya concentrados, los cuales se ocuparon de publicitar las ventajas extraordinarias que generaría la sojización para instalarla en la "agenda publicada" sin entrar en otras consideraciones que la rentabilidad. El tratamiento en términos de agenda pública hubiera sido diferente.

La decisión de "apostar" a la biotecnología para aumentar la productividad fue tomada por el entonces Secretario de Agricultura, Ganadería y Pesca. Desde la perspectiva del periodista de investigación y actual presidente del prestigioso Centro de Estudios Legales y Sociales (CELS), Horacio Verbitsky, se trató de una resolución cuestionable porque no se realizaron las evaluaciones necesarias. Al respecto Verbitsky expresaba en 2009 que el entonces Secretario de Agricultura, Ganadería y 
Pesca, el ingeniero agrónomo Felipe Sola “...también autorizó la introducción en la Argentina de la soja transgénica de Monsanto y el plaguicida que la acompaña, el glifosato. El avance de ese paquete tecnológico amenaza la soberanía alimentaria del país, afecta la calidad de los suelos y ha provocado graves problemas de salud a las poblaciones fumigadas con el agrotóxico que la transnacional estadounidense comercializa con la marca Roundup. La Argentina fue el segundo país del mundo en autorizar la soja RR (por resistente al Roundup), luego de Estados Unidos, en tiempo record y sin otras pruebas que las realizadas por la propia empresa." Solá firmó dos resoluciones: la I I5, del I4 de marzo de 1996, estableció el método de solicitud de autorizaciones para "la experimentación - liberación de la semilla de soja transgénica". La 167, del 3 de abril de 1996, autorizó a producir y comercializar la semilla y los productos y subproductos de la soja "tolerante al herbicida glifosato". Evidentemente, en un mes difícilmente se podía haber experimentado. Tanto es así que Verbitsky hace referencia a varios funcionarios y expertos, quienes plantearon la necesidad de realizar estudios interdisciplinarios para evaluar el impacto ambiental y social. Por ejemplo, para uno de ellos "las autorizaciones deberían emanar del Ministerio de Salud, ya que es imposible obviar los efectos de los transgénicos sobre el ecosistema, sobre la selección natural y sobre otras especies, que no pueden evaluarse en un par de años y sin una investigación multidisciplinaria, compleja y cara."10 Es decir, que hubo voces expertas que plantearon la necesidad de realizar una investigación interdisciplinaria para evaluar posibles consecuencias perjudiciales previsibles. La envergadura de la investigación no debería ser un obstáculo, atendiendo a la magnitud de las transformaciones que se generarían, cuestión que retomaremos en el próximo apartado. Por lo tanto, no se trata de un planteo de historia contrafáctica. Por el contrario, se trata de considerar cómo se deben tomar las decisiones. Parece poco razonable que en el contexto de un Estado constitucional de derecho las decisiones las tome un funcionario de manera discrecional, sin contar con toda la información necesaria y la opinión de los diferentes afectados.

\section{La posibilidad perdida de realizar un estudio interdisciplinario.}

Que no se haya realizado un estudio interdisciplinario de impacto ambiental y social sólo se explica porque se quería implantar la sojización mediante una política de shock que no diera lugar ni a la reflexión, ni a la discusión en el espacio público ni en las instancias legislativas de gobierno. El fenomenal estudio sobre la sorgorización en México que realizara un equipo de la FAO a cargo de Rolando García, muestra que se podía realizar ese estudio ex ante y, que de haberse realizado, se habrían evitado muchas consecuencias indeseables previsibles.

I0 Horario Verbitsky publicó en el diario Página 12 una serie de artículos sumamente documentados de los que resulta imposible dar cuenta en el espacio disponible. Sin embargo, consideramos que su lectura es ineludible si se pretende conocer el modus operandi de algunos lobbies. Los artículos mencionados son: "El predador", el 5 de abril de 2009 (http://www.paginal 2.com.ar/diario/elpais/ subnotas/ I 22647-39235-2009-04-05.html); "Verano del '96", publicado el 26 de abril de 2009 (http://www.pagina I 2.com.ar/diario/ elpais/ I - I 23932-2009-04-26.html) y "El estudio de campo", del 10 de mayo del mismo año (http://www.paginal2.com.ar/diario/ elpais/ I- | 24649-2009-05- | 0.html) donde a través de una entrevista el ingeniero Felipe Solá ensaya una defensa de lo actuado. 
Efectivamente, Rolando García elaboró una metodología interdisciplinaria a partir de la investigación ex post sobre la implantación de un paquete tecnológico para la sorgorización (plantación intensiva de sorgo) de una región de México, el Valle de Santiago en el Estado de Guanajuato." Si bien no podemos dar cuenta en este espacio de esa metodología, sí tenemos que hacer referencia a algunos aspectos.

En primer lugar, corresponde destacar que lo que posibilita el "inter" de la interdisciplina es la definición de un marco epistémico previo, compartido por los científicos de las diferentes especialidades. Ese marco epistémico no es meramente un marco teórico, sino que tiene componentes valorativos que sirven como criterios para distinguir lo deseable de lo indeseable. Actualmente, en buena medida, esos valores están plasmados en los pactos internacionales sobre derechos fundamentales, cuyo reconocimiento internacional nos exime de una mayor fundamentación en el presente contexto. Cabe destacar que, a su vez, esos pactos internacionales permitieron elaborar la norma ISO 26000, la cual debería ser tenida en cuenta por las organizaciones públicas y privadas que tengan alguna pretensión genuina de ser responsables social y ambientalmente. Particularmente el "Principio de debida diligencia". ${ }^{2}$

El marco epistémico, en consecuencia, orienta la investigación hacia su propósito final, a saber, revertir o, al menos, detener el daño causado, en este caso por la sorgorización. Se trata de una investigación del tipo de los "grandes programas" que se plantea proponer un esquema de desarrollo alternativo que sea sustentable, es decir, que satisfaga las necesidades de las actuales generaciones sin comprometer las posibilidades de las futuras generaciones para satisfacer sus propias necesidades.

En segundo lugar, debemos destacar, con respecto a la metodología, que García explica que en el sistema complejo estudiado existen procesos de diferentes niveles que no deben confundirse si se quiere comprender correctamente la interacción entre ellos. Hay niveles "superiores", en el sentido de ser más abarcativos, que condicionan y a veces sobredeterminan a los niveles inferiores.

En el primer nivel o nivel de base se realiza un estudio de diferentes subsistemas, los cuales podemos simplificar en subsistemas físico, social y económico. El aspecto físico, es distinguible a su vez en el suelo, que puede incluir la calidad de la tierra o su deterioro por salinización; el agua, la cuenca hídrica, el nivel de la napa freática; el aire, los aspectos climáticos; los aspectos biológicos. Por su parte, las formas de producción, de intercambio, de acumulación y de tenencia de la tierra requieren ser estudiadas por la economía. A su vez, la economía debe atender a los antecedentes históricos y culturales, entre otros elementos. La integración social y el trabajo serán estudiados por la sociología y así sucesivamente.

I Estas cuestiones fueron presentadas en dos artículos: "Conceptos básicos para el estudio de sistemas complejos" García, Rolando, en Enrique LEFF, Los problemas del conocimiento y la perspectiva ambiental del desarrollo, México, Siglo XXI, I 986 y "La investigación interdisciplinaria de sistemas complejos", publicado originalmente por el Centro de Estudios Avanzados - UBA - Serie Materiales |/91, 199|. Ambos trabajos fueron recogidos prácticamente sin modificaciones en un libro que compila también otros artículos de García: Rolando García, Sistemas complejos, Conceptos, métodos y fundamentación epistemológica de la investigación interdisciplinaria, Barcelona, Gedisa, 2006.

12 A la Norma ISO 26000 nos referimos en "Las posibilidades de una ética cívica empresarial en la era de la globalización", capítulo 8 del libro Ética, política y mercado, ya citado. 
En el segundo nivel aparecen cuestiones vinculadas con cambios tecnológicos que van a determinar el primer nivel, los cuales también requieren del estudio de la economía política.

Si bien la conflictividad puede darse en todos los niveles, quizás el tercer nivel sea el más político en el sentido de la definición de políticas por parte de los Estados, pero también en cuanto a la interacción de los Estados entre sí y con respecto al mercado, constituido por corporaciones con enorme capacidad de incidencia política en las reglas de juego, con incidencia mediática directa y, por supuesto, con incidencia económica. Supone las políticas nacionales de desarrollo, las reglas de juego del mercado internacional, la internacionalización de capitales, etc., los cuales condicionan fuertemente la dinámica de los procesos de segundo y primer nivel.

Los desequilibrios que se generan en el nivel I, pueden explicarse por las políticas generadas en el nivel 3, posibilitadas por las tecnologías incorporadas en el nivel 2.

En este punto, si quienes definen las políticas realmente se hacen responsables social y ambientalmente, se requiere la constitución de un equipo interdisciplinario que busque una respuesta adecuada en términos de -según sea el caso o el momento- prevenir, revertir o al menos detener los daños que se generan. Atendiendo a lo ya explicado, la interdisciplina no consistirá en la mera coordinación de resultados de los estudios de las diferentes disciplinas, sino que será un largo proceso con etapas de integración y etapas de diferenciación. Integración por aproximaciones sucesivas de los conocimientos logrados en cada uno de los subsistemas en los que se diferencia el sistema dinámico complejo.

\section{I La sorgorización en México}

Veamos algunos resultados del estudio que dirigió Rolando García en México para plantear la analogía con la sojización en Argentina. A la región del Bajío se la conocía como "el granero de México", importante productor de trigo y maíz. En la década de 1950 se introdujo el sorgo, el que fue presentado como el cultivo ideal por el suelo y el clima. Además con un alto precio garantizado como forraje por el desarrollo de la ganadería. Al respecto expresa García: "El cultivo de sorgo contaba con el apoyo de un sistema de crédito que incluía un paquete tecnológico con semillas mejoradas, nuevos tipos de fertilizantes químicos, control de plagas y herbicidas (...) El crecimiento de la demanda de sorgo y el éxito económico de sus productores estimuló un nuevo avance del cultivo durante los años 1970. El mecanismo era el mismo: la monopolización de la tierra y la perforación de pozos."'3

García da cuenta de las consecuencias sociales indeseables: desplazamiento de campesinos, migración, desempleo, proletarización, empobrecimiento, desnutrición. En cuanto a las consecuencias ambientales indeseables: explotación no controlada del agua del subsuelo, el manto freático bajó de ocho metros en 1955 a 200 metros en 1982. El agua de ese nivel es salinizada y salinizó la tierra. Las

13 García, Sistemas complejos, pág. 174. 
nuevas máquinas compactaron y endurecieron el suelo, lo cual llevó a utilizar máquinas todavía más pesadas. Se trata sin dudas de una forma de crecimiento predatorio con deterioro social y ambiental.

\subsection{La sojización en Argentina. La ausencia adrede de una investigación interdisciplinaria}

Como veremos, el estudio sobre la sorgorización en el Bajío de México que encargara la FAO a Rolando García guarda claras analogías con el proceso de sojización en Argentina. Llama la atención que quienes en el nivel político tomaron en su momento las decisiones que posibilitaron un proceso de sojización características predatorias no hayan tenido en cuenta estos estudios, ya disponibles por entonces y que, por la formación del funcionario de marras, deberían ser conocidos. Evidentemente, no se quisieron tener en cuenta, porque siempre fue muy fuerte la capacidad de lobbying sobre el Estado mínimo, funcional a los mercados autorregulados, característico de los años ' $90.1^{14}$

Una implementación de la metodología interdisciplinaria desarrollada por Rolando García para un estudio interdisciplinario de la sojización en Argentina supondría definir el sistema complejo a estudiar y el marco epistémico que orientará la indagación, en vista al planteo de una estrategia de desarrollo alternativa. Atendiendo a la magnitud que adquirió el proceso de sojización de la economía en nuestro país desde mediados de los años '90, parece claro que -aunque se está corriendo permanentemente la frontera agrícola - se puede recortar el sistema desde una perspectiva geográfica atendiendo al impacto ambiental en la región pampeana, el litoral y el NEA (nordeste argentino). Sin embargo, el impacto social, económico y político afecta a todo el país. Por su parte, el marco epistémico requiere clarificar los elementos valorativos compartidos que orienten la investigación -atendiendo a una política alternativa- en términos de sustentabilidad ambiental y social, considerando no sólo el bienestar o los perjuicios de las actuales generaciones, sino también de las generaciones futuras. En este caso, para simplificar la cuestión en torno a los valores involucrados, consideramos que se debe recurrir a los valores plasmados en torno al equilibrio entre los derechos fundamentales, incluidos los derechos económicos, sociales, culturales y ambientales, dado que son reconocidos por unos 170 países y en Argentina forman parte de la Constitución Nacional.

Con respecto a la estratificación de los procesos, encontramos condicionamientos y consecuencias que se pueden analizar en los diferentes niveles considerados por García.

I4 Al respecto nos referimos en diferentes trabajos, entre ellos en Javier Flax, "La implantación del neoliberalismo mediante la concentración y abuso de poder, Capítulo 4 de Ética, política y mercado. En torno a las ficciones neoliberales, Los Polvorines, UNGS, 20 I3, del cual el capítulo 5 podría brindar algunas claves de lectura. También en "El decisionismo revisitado. Un contrapunto entre los gobiernos de Menem y Kirchner", Revista Diálogo Político, Fundación Konrad Adenauer, Año XXVIII, N², junio de 201 I. Ahora bien, la denominada dictadura de la deuda externa y la dependencia de los organismos multilaterales de crédito puede explicar la sensibilidad de los funcionarios durante la presidencia de Carlos Menem, sin necesidad de suponer que haya habido meramente corrupción. 
Los procesos llamados básicos o de primer nivel, constituyen, generalmente, el efecto local sobre el medio físico o sobre la sociedad que lo habita y lo explota. Ejemplo: a) Efectos físicos: efectos de la sojización sobre el suelo, deforestación, tala de bosques para correr la frontera agrícola; erosión y pérdida de nutrientes de la tierra (pérdida de hidrógeno y fósforo, éste último no renovable), siembra directa mediante el uso de herbicidas como el glifosato. ${ }^{15}$ b)efectos sociales y sobre las personas: consecuencias del glifosato en los organismos, patologías correlacionadas con el glifosato, malformaciones, cáncer. ${ }^{16}$ Desplazamiento de la población y la pérdida de trabajo agrícola a partir del cambio en los modos de producción, mediante la incorporación de nuevas tecnologías para la siembra y cosecha que conducen a c) efectos económicos: encarecimiento del valor de la tierra para otros tipos de producción, con el consecuente aumento en el precio de los productos básicos para la alimentación de nuestra población.

En el segundo nivel se encuentra claramente el paquete tecnológico constituido por la soja transgénica RR (resistente al Roundup), el propio glifosato (Roundup) y las maquinarias para implementar

I5 Cabe mencionar que si la técnica de siembra directa preservara efectivamente mejor a la tierra, en estos tipos de explotación ese cuidado se ve menoscabado por la deforestación y por la falta de rotación de los cultivos, sostenida o exigida solamente por aquellos productores o arrendadores más conscientes. Por otra parte, la absorción del agua se complica y los canales abiertos generan rápidamente inundaciones en los momentos de lluvias intensas.

I6 Ya dejaron de ser controversiales las consecuencias patogénicas del glifosato. En nuestro medio, el vapuleado Dr. Andrés Carrasco avanzó en mostrar efectos teratogénicos y genotóxicos del glifosato, en estudios realizados por el en el Laboratorio de Embriología Molecular, CONICET-UBA, Facultad de Medicina, Universidad de Buenos, a partir de lo cual comenzó a ser perseguido.

A partir de la resistencia de la población de la localidad de Malvinas Argentinas, Córdoba, a que se instale allí una planta de Monsanto, la agencia de noticias The Associated Press, realizó una investigación que publicó en un artículo sumamente documentado sobre las consecuencias del uso del glifosato en Argentina. Frente a esa información rigurosa, el portavoz de Monsanto expresó con respecto a la salud humana "la ausencia de datos confiables dificulta mucho establecer tendencias en la incidencia de enfermedades y todavía más difícil establecer relaciones causales. En nuestro conocimiento, no hay relaciones causales establecidas". Lo que Monsanto declaró es lo mismo que ocurriera tantas veces con otros productos patogénicos de la industria, como el cigarrillo. Las que están muy claras son las correlaciones establecidas entre el uso del herbicida Roundup, cuya principal componente es el glifosato diferentes daños a la salud humana, como lo indican estudios de asociaciones médicas. En Santa Fe, las tasas de cáncer son entre dos y cuatro veces más altas que el promedio nacional. En el Chaco, los defectos de nacimiento se cuadruplicaron desde hace 17 años cuando el uso de la biotecnología aplicada al campo se disparó. Desde 2010 en Santa Fe y Córdoba hay fallos judiciales firmes que ponen límites al uso del glifosato e, incluso, se condenó a prisión a productores y fumigadores. Entre 201 I y 2012 la Auditoría General de la Nación realizó una investigación en torno a la problemática, cuyo informe se aprobó en diciembre de 2012, recomendando que el Estado, a través de sus organismos realice un control efectivo de los plaguicidas. El vocero de Monsanto, frente al informe de la agencia The Associated Press evidenció un cambio en la estrategia discursiva de la empresa: "Si los pesticidas están siendo mal utilizados en Argentina, entonces es en el mejor interés de todos, el público, el gobierno, los agricultores, la industria y Monsanto, que se detenga su mal uso". Es decir, se pasó de sostener la inocuidad para la salud humana a asignar las consecuencias patogénicas al mal uso por parte de los productores y a poner actualmente la carga de la publicidad en un buen uso. Sin embargo, ya en 1996 Monsanto había sido multada en USA y aceptó dejar de publicitar el glifosato como "seguro", después de que un fiscal en Nueva York demandara a la empresa por falsa publicidad. Lo cual no significa que, además de los "efectos laterales" del propio glifosato, estos no se potencien por un uso indiscriminado. Fuente: "Argentines Link Health Problems to Agrochemicals", by Michael Warren and Natacha Pisarenko, The Associated Press, Oct. 20, 2013.

Las preguntas que surgen son las siguientes, ¿el impacto del glifosato, e incluso el mal uso del glifosato no podía haber sido previsto?, ¿no debía al menos haberse aplicado el "principio de precaución" de acuerdo con el cual ante la falta de certeza sobre los riesgos, es preferible abstenerse?. 
la siembra directa a gran escala. También se puede incluir en este nivel el cambio en la modalidad de explotación de los pequeños y medianos propietarios a quienes resulta más rentable arrendar sus tierras a los grandes pooles tecnificados. Para obtener una mayor rentabilidad, conviene la siembra de soja en una escala mayor y utilizar maquinarias sumamente caras. Para ello se cuenta con el financiamiento de los pooles sojeros, organizados en torno a inversionistas que quieren obtener la mayor rentabilidad por su dinero. Hoy es la soja, mañana vaya a saberse qué, si cae el precio de la soja. ${ }^{17}$

En el tercer nivel se pueden considerar metaprocesos más amplios -o procesos que tienen lugar en otros niveles: mercados abiertos, precios de un commodity como la soja, valorizada por las mejores condiciones de alimentación de economías emergentes como la China e India. En este nivel no puede desatenderse los ingresos que genera la sojización al país, no sólo a los sectores concentrados del agro, sino al erario público en términos de retenciones a las exportaciones. Tampoco puede desatenderse que atiende la necesidad alimentaria de la población de los países mencionados, los que utilizan la soja para la cría de animales que utilizarán como alimento. Sería parte de la investigación interdisciplinaria determinar si es el único modo factible de paliar el hambre de esas poblaciones. ${ }^{18}$

El impacto del tercer nivel sobre los niveles anteriores no es automático, sino que depende de la política económica. No es lo mismo un Estado activo monitoreando y regulando, que un Estado que meramente deja hacer a los mercados. En nuestro país se impusieron políticas neoliberales de "mercados autorregulados" con apertura de la economía y desregulaciones. Se puede considerar que la autorización de los eventos biotecnológicos y la falta de evaluación de las consecuencias indeseables en función de las ganancias inmediatas fueron posibilitadas por un Estado sensible al poder del mercado. Podría existir una mayor "clausura" del sistema económico argentino, pero la apertura indiscriminada de los mercados eliminó esa posibilidad. En el caso argentino, la renuncia del Estado en los '90 a orientar la economía y la pérdida de capacidad estatal para intervenir, condujo a que en nuestro país el fenómeno de la sojización adquiriera características alarmantes, incomparables con otros países. La soja era un cultivo poco representativo y se fue expandiendo en detrimento de otras producciones. ${ }^{19}$

17 El precio efectivamente viene cayendo, como se había previsto, no tanto por caída de la demanda como por la caída del precio a futuro en la Bolsa de Chicago, atendiendo a que el precio tenía un importante componente especulativo. De unos U\$S 580 la tonelada, bajó a U\$S 380 aproximadamente en pocos meses.

18 Debe tenerse en cuenta que las cifras actuales de la FAO establecen que el 25\% de la población mundial, particularmente en Asia y África padece hambre. En América Latina se estima que 57 millones de personas padecen hambre. Pero se considera que existen otras alternativas productivas en la línea de la agroecología, la cual es actualmente fomentada por la misma FAO. Si se continuara con transgénicos resistentes al glifosato, deberían tomarse todas las precauciones necesarias, las cuales resultarían de un estudio interdisciplinario como el propuesto.

19 Para ver la evolución gráficamente, sugerimos ver un mapa dinámico sobre la superficie sembrada elaborado por el CONICET en http://www.laargentinaenmapas.com.ar/caste/soja/soja_se.htm Para tener una idea más cabal, se pueden observar los mapas evolutivos (o involutivos) referidos a otros cultivos, en términos de superficie, producción y rendimiento por hectárea. 


\subsection{En torno al desempleo, al desplazamiento poblacional y otros daños}

Los estudios sobre la necesidad de mano de obra para los cultivos realizados mediante organismos genéticamente modificados muestran una sensible disminución en la necesidad de mano de obra. Por ejemplo, si un cultivo tradicional de algodón requiere 180 horas/hombre por año por hectárea, la misma superficie sembrada con soja transgénica requiere solamente 4 horas/hombre. Parecería que evaluar las consecuencias indeseables sobre el empleo rural no resulta tan complicado. El resultado fue la migración forzosa de los campesinos -muchos pequeños propietarios- muchas veces mediante expulsión violenta, hacia el conurbano de las grandes ciudades. En el año 2008, el entonces gobernador de la provincia argentina de Santa Fe, Hermes Binner, expresaba: "Si nos dejamos llevar por el precio de la soja, en una provincia como Santa Fe, en poco tiempo no va a quedar nada de las demás actividades productivas. El proceso de sojización ha significado vaciar el campo, que la gente migre a las ciudades, con oficios que no corresponden a las demandas que hoy tiene una ciudad, a vivir en los peores lugares, lo que genera planes sociales que nunca tendrán el valor del trabajo como elemento formador del individuo y la sociedad." ${ }^{20}$ En otro momento, el mismo gobernador expresó que "necesitamos un proyecto donde no se discuta si es más importante la soja o los pobres" (Discurso del 2/4/2008). Por supuesto, que plantearlo en esos términos sería incurrir en un pseudo dilema, porque debería haber habido desde el principio una previsión de cómo continuaría la vida de los peones rurales y de los campesinos en general. ¿Podía preverse el desplazamiento poblacional y las dificultades de desarraigo e inserción laboral de los desplazados? Claramente sí. Si en el contexto del neoliberalismo se apostó a un proyecto de desarrollo agrícola basado en las biotecnologías, debería existir la disposición de los grandes ganadores de ese modelo a resignar parte de su renta extraordinaria para compensar a quienes terminaron siendo perdedores de todo. Sin embargo, son reacios a pagar las retenciones a las exportaciones con las que el Estado financia la política social.

Es así que los ingresos que tiene el Estado en calidad de retenciones a las exportaciones, se van por la puerta de la ayuda social a los desplazados. Efectivamente, la sojización transgénica le generó al Estado argentino ingresos sumamente importantes en una situación de recesión económica. Más aún, a partir del establecimiento de retenciones a las exportaciones, el fisco logró ingresos que llegaron a significar hasta el 10\% del presupuesto nacional. Lo que no está claro es cuánto de esos ingresos se tuvo que destinar a mitigar las consecuencias de la misma sojización. Efectivamente, las retenciones a las exportaciones agropecuarias se implementaron para que los precios internos de los alimentos no treparan, luego de una enorme devaluación del peso argentino, al salirse de la convertibilidad entre el peso y el dólar estadounidense. Esas retenciones fueron paulatinamente más significativas para las arcas públicas, conforme aumentaba la siembra de soja y el precio internacional de la misma. Probablemente por esa razón los gobiernos nacionales no quisieron aún ocuparse de las consecuencias más evidentes de la sojización. La misma población desplazada o rociada con glifosato es actualmente objeto de la política social -incluida la salud pública- a través de diferentes iniciativas que deberían cuantificarse

20 Hermes Binner, diario La Nación 1\%/02/2008. 
en términos de erario público. Esas políticas son valiosas, aunque epimeteicas, y no pueden mitigar el desarraigo de la gente ni muchas consecuencias indeseables propias de procesos migratorios no planificados. Analógicamente, como ocurría con el tabaco, a pesar de la carga impositiva significativa que tiene en Argentina el precio de los cigarrillos, lo recaudado apenas alcanza para que el Estado se haga cargo, mediante el generoso sistema de salud pública argentino, de las patologías generadas. Pero los padecimientos de los enfermos y sus familias no se mitigan con esos recursos pecuniarios.

Ante la omisión del Poder Ejecutivo de una política de prevención y protección, varios jueces tuvieron en cuenta las correlaciones entre el uso del glifosato, el aumento de las malformaciones genéticas y la incidencia del cáncer en las poblaciones afectadas, denunciadas por las asociaciones médicas. Así fue que se lograron sentencias para limitar el uso del glifosato, primero, y luego a productores y rociadores que no acataron sus fallos. Finalmente, el 20 de marzo de 20 I 5, tardíamente, la IARC (Internacional Agency for Reserach on Cancer), dependiente de la OMS procedió a categorizar el herbicida glifosato como 2A, es decir, como un cancerígeno probable.

Cabe entonces preguntar: ¿cuál es la ganancia en términos de interés público por los ingresos al fisco por retenciones a las exportaciones de soja?

\section{La economía global y las reglas de juego internacionales en torno a las patentes}

Como expresamos, debe incluirse entre los procesos de segundo nivel la introducción de semillas transgénicas por parte de laboratorios multinacionales que se erigen en proveedores oligopólicos de semillas certificadas y de glifosato. En el caso de la soja transgénica, el primer evento autorizado es la soja RRI, que significa "resistente al Roundup", marca comercial del laboratorio Monsanto del glifosato, cuya patente le pertenecía, pero caducó. El glifosato es necesario para la siembra directa porque elimina todas las malezas, salvo las resistentes al Roundup. Por lo cual, sin glifosato no puede haber sojización transgénica ni siembra directa. Sin dudas, acá está el quid de la cuestión: proveer de manera mono y oligopólica semillas transgénicas patentadas y desde allí controlar todo el negocio.

Como expresa Joseph Stiglitz en línea con otros estudiosos, las patentes, lejos de fomentar la innovación científica y tecnológica, erigen barreras a la investigación y constituyen monopolios artificiales, basados en las reglas de juego en torno a esa modalidad de la propiedad intelectual. ${ }^{21} \mathrm{Al}$ respecto expresa Stiglitz:

21 Stiglitz, Joseph Cómo hacer que funcione la globalización, Buenos Aires, Taurus, 2006, capítulo "Patentes, beneficios y personas". Es también importante tomar en cuenta el capítulo "La corporación multinacional" del libro citado, Stiglitz muestra que los gobiernos de los países desarrollados actúan en los organismos internacionales para proteger a esas corporaciones. Su lectura permite comprender de qué manera las corporaciones logran que gobiernos como los de USA se pongan al servicio de la defensa de sus patentes y de sus utilidades. Por ello dice Stiglitz que "el liberalismo es proteccionista de sus corporaciones, con lo cual las asimetrías se agudizan", Op. Cit., pág. 246. 


\begin{abstract}
"Algunos críticos han comparado el reciente refuerzo de los derechos de propiedad intelectual con la iniciativa de levantar cercados que en la Baja Edad Media se produjo en Inglaterra y en Escocia, cuando los señores feudales empezaron a cercar tierras que hasta ese momento habían pertenecido a toda la comunidad. Pero existe una importante diferencia con lo que hoy está ocurriendo: aunque las personas expulsadas de sus tierras sufrían enormemente, la eficacia económica experimentaba cierta mejora (...) Los economistas dirían que se trata de un clásico caso de sacrificio de equidad por eficiencia. Pero en el cercado de los territorios comunes de lo intelectual, sí se pierde eficiencia." 22
\end{abstract}

Está demostrado que diferentes investigadores o equipos de investigación pueden llegar a los mismos resultados, pero las reglas de juego impuestas hacen que el que llega primero se quede con los derechos exclusivos, lo cual desalienta la investigación. Atendiendo a que se utilizan conocimientos públicos y que diferentes investigadores pueden llegar a los mismos resultados, se requiere poner un límite al derecho a la propiedad intelectual bajo la forma de patentes para aquellos casos en los que realizan patentamientos en torno a conocimientos que se orientan a la satisfacción de necesidades básicas, como los alimentos y los medicamentos. Lejos de ello, el Acuerdo sobre ADPIC reforzó el esquema que erige cercados en torno al conocimiento.

Pero hoy por hoy las patentes se utilizan efectivamente para erigir monopolios. En el caso estudiado, para controlar el negocio de la alimentación a nivel mundial, cuando la alimentación es ante todo una necesidad básica y un derecho fundamental. En estos casos, debe priorizarse el derecho al conocimiento y a la satisfacción de las necesidades básicas por sobre el derecho a la propiedad bajo la forma de patentes, la cual generalmente significa una apropiación y una privatización de conocimientos públicos.

Como expresa un especialista argentino, el Dr. Aldo P. Casella, el sistema de patentes se construyó para proteger la propiedad industrial, particularmente para los productos mecánicos y por ello quedaron excluidos las especies y los productos vegetales. Sin embargo, se lo fue extendiendo impropiamente a otros campos, hasta llegarse al colmo de patentar la vida, la cual, a diferencia de los productos mecánicos es autoreproducible. Asimismo, los propios agricultores fueron realizando los procesos de mejora de las semillas, seleccionando en cada cosecha aquellas mejores para las cosechas siguientes, durante miles de años. E incluso, generando variedades nuevas, siendo la base del trabajo de los actuales fitomejoradores. "Ya avanzado el siglo pasado -expresa el Dr. Casella- los fitomejoradores profesionales y las empresas especializadas en semillas promovieron la protección intelectual de sus nuevas variedades, se concibió un sistema propio, distinto y posterior al sistema de patentes, denominado Derecho del Obtentor Vegetal (DOV)". ${ }^{3}$ En nuestro país la legislación

22 Op. Cit, pág. 150.

23 Casella, Aldo P. "Argentina: Ley de semillas, propiedad intelectual y políticas públicas", publicado en http://www.biodiversidadla.org/ Portada_Principal/Documentos/Argentina_Ley_de_semillas_propiedad_intelectual_y_politicas_publicas. El Dr. Casella es profesor, investigador, especialista en derecho agrario de la Nacional del Nordeste y la Universidad Nacional del Litoral. 
permite el libre uso de las semillas y de los productos. Pero las compañías trasnacionales pretenden asimilar el régimen al de las patentes, con el objeto de cobrarles en cada siembra a los agricultores por el uso de sus semillas, del mismo modo que lo lograron en algunos países mediante Tratados de Libre Comercio, totalmente asimétricos por la envergadura de los países y por los condicionamientos abusivos correspondientes. ${ }^{24}$ Como expresa el Dr. Casella, en el caso del algodón RR, además del monopolio de las semillas, su posición de dominio en el mercado les permite designar las desmotadoras, cooperativas, empresas de acondicionamiento y tratamiento de semillas y/o semilleros y multiplicadores que prestarán los servicios de desmote, multiplicación, acondicionamiento, tratamiento y distribución del producido de la semilla de algodón. Es decir, a partir de sus derechos intelectuales sobre la "tecnología", dominarán toda la cadena productiva y comercial del algodón."

\section{Opacidad y lobbying en torno a las decisiones}

Volviendo a la autorización de la soja RRI en Argentina, como expresan estudiosos del tema, responde más a un interés externo que a un interés interno. Este interés consiste en "la constitución de "plataformas productivas" en los países del sur, que se orientan a satisfacer las estrategias globales de abastecimiento de las grandes corporaciones." 25 Por supuesto, se puede partir del principio de presunción de inocencia o de la buena fe de los funcionarios y expertos y considerar que la globalización condujo a un nuevo esquema internacional de división del trabajo, en el cual nuevamente a la Argentina le tocaría el papel de ser mero productor de materias primas, dado que tiene ventajas comparativas - en términos de la discutible teoría del economista David Ricardo- para ser eficiente en ese tipo de producción. Entonces se trataría de una convergencia de intereses. Ahora bien, los países deben aprovechar sus ventajas comparativas, pero no a costa de renunciar a un desarrollo integrado y sustentable.

De cualquier manera, en una democracia constitucional las políticas de fondo no pueden ser decididas de manera discrecional por un funcionario. Ni puede sostenerse que la decisión es el resultado de la racionalidad de un grupo de expertos en una comisión constituida de manera sesgada para justificar esa decisión, como la CONABIA (Comisión Nacional Asesora de Biotecnología Agropecuaria).

24 Algo similar está ocurriendo en otros países de América Latina con semillas bajo propiedad intelectual. En Colombia, por ejemplo, el acuerdo bilateral de libre comercio con USA condujo a la Resolución 970/20I0 del Instituto Colombiano de Agricultura (ICA) por la cual por "razones sanitarias" sólo se podían usar semillas certificadas. Por ello efectivos y camiones del ejército se ocuparon de decomisar y destruir las semillas que los campesinos guardaron de la cosecha anterior.

Es tan liberal el "libre comercio" que se aplica manu militari y los agricultores pierden la libertad de conservar sus propias semillas y elegir su estilo de agricultura, lesionándose derechos fundamentales. Si existiera una intención genuina de garantizar la seguridad sanitaria, debería el Estado implementar una oficina de certificaciones, antes que obligar a comprarle semillas a proveedores oligopólicos, probablemente cartelizados. El producto de la tierra ya no es ni para sus dueños, ni para quien la trabaja, sino para quienes tienen la propiedad intelectual de las semillas sea como "obtentores" o como patentadores y son proveedores monopólicos. Un clásico ejemplo de re-regulación neoliberal que avanza además contra la biodiversidad. Ojalá la resistencia a estos abusos brinde sus frutos si se revierte efectivamente la resolución 970.

25 Carla Gras y Valeria Hernández "Los pilares del modelo agrobusiness", en Carla Gras y Valeria Hernández (comps.) El agro como negocio, Buenos Aires, Biblos, 2013, pág. 22. 
Quienes investigaron específicamente la actuación de la comisión expresan que si no fue integrada directamente mediante la influencia del lobby empresarial encabezado por Monsanto, son evidentes las características tecnocráticas y cientificistas de la comisión. ${ }^{26}$ Cientificista en el sentido de Oscar Varsavsky y tecnocrática por las pretensiones de que una racionalidad técnica e instrumental puede determinar objetivos valiosos que la exceden. En la comisión no se dio lugar a otras perspectivas que no fueran las de una racionalidad técnica de expertos con una visión meramente productivista. Por lo tanto, no evaluaron otros aspectos que las ventajas económicas de los eventos biotecnológicos con el único fin de legitimar una política agropecuaria, que hubiera sido considerada indeseable en muchos aspectos si la comisión hubiera sido interdisciplinaria o abierta al escrutinio público. Por otro lado, si se supone que esa racionalidad supone que se tiene que construir un conocimiento "objetivo, válido, fiable y completo", por lo menos el conocimiento aportado no fue completo. Se dejó afuera sistemáticamente a aquellos científicos que podían completar ese conocimiento: "La imposibilidad de "hablar el mismo idioma" generó que aquellos organismos gubernamentales que tenían una mirada asociada al "desarrollo sostenible" (Secretaría de Medio Ambiente) o aquellos cientíicos que planteaban una visión ampliada del análisis de riesgo no tuvieran incentivos para participar en la comisión." 27

Debemos insistir en que la racionalidad científica y técnica en tanto tal, no tiene capacidad para realizar una evaluación de prioridades en términos de valores, porque éstos exceden esa racionalidad. En consecuencia, los componentes valorativos suponen instancias de interpretación política o filosófico-jurídica tomando como base los derechos fundamentales que, en el caso de la República Argentina, desde la reforma de la Constitución Nacional en 1994, constituyen leyes constitucionales. Pero estas instancias políticas se eludieron sistemáticamente. "La permanencia del discurso experto, en los términos previamente analizados, continuó evitando las miradas "politizadas" de aquellos que podían tener alguna opinión asociada al ordenamiento territorial, al cuidado del ambiente o a la información al consumidor." ${ }^{28}$ En ese sentido, la totalidad de la decisión política quedó reservada a la discrecionalidad de un funcionario del Poder Ejecutivo, el Secretario de Agricultura, Ganadería y Pesca, quien concentró la decisión en un contexto de opacidad o falta de transparencia frente a otros poderes del Estado y de la opinión pública en general. En todos estos años, lo que viene ocurriendo es que estos asuntos son sistemáticamente sustraídos al tratamiento público y a las instancias de representación política legislativas que pudieran dar lugar a un debate público que cuestione las políticas o exija salvaguardas para evitar consecuencias perjudiciales previsibles que aumentaran los costos de producción si se pretendía evitarlas. Por el contrario, las discusiones se dan en lugares ina-

26 Sobre el modo non sancto de operar de Monsanto existe una vasta bibliografía, comenzando por el ya clásico libro y el documental de Marie Monique Robin, El mundo según Monsanto en el que muestra la estrategia agresiva de esa compañía para fomentar el empleo de transgénicos y agroquímicos, sin advertir sobre las consecuencias para el suelo y las personas. O peor aún, "manipuló estudios científicos para tapar los efectos de las toxinas sobre el ser humano". Ahora bien, debe quedar en claro que no es la única empresa que provee soja transgénica. Empresas argentina recurren a las variedades patentadas por Dupont, la cual recordemos, tuvo un litigio con Monsanto -a la que acusó de prácticas monopolistas- por esas patentes.

27 Carla Poth "Reconstruyendo la institucionalidad del modelo biotecnológico", en Carla Gras y Valeria Hernández (comps.) El agro como negocio, Buenos Aires, Biblos, 2013 , pág. 305.

28 Carla Poth "Reconstruyendo la institucionalidad del modelo biotecnológico", pág. 312. 
propiados como la Embajada de USA. Esta afirmación no es el resultado de una visión conspirativa ni de especulaciones, sino que es lo que muestran los cables publicados por Wikileaks, correspondientes al período inmediatamente posterior. ${ }^{29}$

En el año 2012 se aprobó un nuevo evento, la soja RR2, en una lógica que parece no diferenciarse de la anterior. En este momento hay dos nuevos eventos de Monsanto con dictamen de la CONABIA esperando su aprobación. ${ }^{30}$ El agropower instalado parece no tener límites. Quizás porque se instaló la creencia de que -como afirma un conocido lobbista, en esa analogía completamente falaz- "la Argentina sin soja es como Kuwait sin petróleo". Independientemente de si la resolución se tomó siguiendo los procedimientos formales o no -lo cual no es menor- el problema, insistimos está en la sustracción al debate público de una decisión que involucra de diferentes modos al conjunto de la población.

Con la soja RR2 los principales perjudicados -entendemos nosotros- van a ser los productores, dado que muy probablemente se les va a dificultar comprar las "bolsas blancas" de semillas transgénicas de soja RRI sin pagar regalías en concepto de patentes, sino que van a tener que pagar mucho más por las semillas de soja RR2. Como puede leerse en el artículo "Ser o no ser, esa es la cuestión", publicado en el suplemento Clarín Rural el 23 de febrero de 2013, se ponen de manifiesto las bondades de la soja Intacta RR2, como más resistente a determinadas plagas, como con una mayor capacidad de rinde. Lo que queda claro es que el principal objetivo de este evento o versión de soja transgénica es la trazabilidad al efecto del cobro de regalías, lo que significa que cuando se usa esa semilla, es posible identificar el origen de la misma en el germoplasma patentado por Monsanto y, en consecuencia, como se ve en el artículo, en cualquier momento del proceso de producción o de comercialización, los productores deberán abonar las regalías a las empresas comercializadoras de la nueva variedad de Monsanto. ${ }^{31}$

29 Nos referimos a los cables de las embajadas que publicara Julián Assange y muestran los entretelones del ejercicio del lobbying. El periodista Santiago O'Donnell publicó ArgenLeaks. Los cables de Wikileaks sobre la Argentina, de la A a la Z, Buenos Aires, Sudamericana, 20l I. En la letra M aparece un extenso artículo -cuya lectura es imprescindible- sobre los cables vinculados con el lobby ejercido por Monsanto sobre el gobierno argentino a través de un sinnúmero de funcionarios norteamericanos -embajadores, senadores, congresistas de la cámara baja, miembros del gabinete norteamericano- con el propósito de cobrar regalías y conseguir una nueva ley de semillas a la medida de sus intereses. Afortunadamente, en el período que comenzó en 2003, los funcionarios tuvieron una posición firme de defensa de los intereses argentinos, salvo algún funcionario de tercera línea que no pudo influir. Finalmente Monsanto retiró las demandas en Europa contra Argentina para lograr la aprobación de la soja RR2. Ibid., pág. 232 y ss.

30 Cabe destacar que la mayoría de los eventos autorizados por la autoridad competente corresponden a eventos de Monsanto. El procedimiento de autorización está regulado actualmente meramente por una resolución del Ministerio de Agricultura, Ganadería y Pesca, la Res. MAGyP N763/20 I I y los organismos intervinientes de ningún modo llegan a realizar un estudio de impacto que contemple todas las cuestiones tratadas en el presente artículo. Basta con recorrer la página web institucional: http://www. minagri.gob.ar/site/agregado_de_valor/biotecnologia/20-CONABIA/index.php

31 "La soja Intacta RR2 Pro, tiene el nuevo gen RR2 (que resiste al glifosato y al mismo tiempo brinda una sensible mejora de rendimiento) junto con un gen Bt que le brinda resistencia a insectos lepidópteros". Más adelante, un gerente de Monsanto explica que "Está claro que la Intacta llega para cambiar el negocio de soja en la Argentina. Y no solo desde lo tecnológico, sino también desde lo organizacional, si se quiere. Por esta tecnología habrá que pagar, a diferencia de lo que sucedió con la RR que hasta hoy se sigue sembrando, por la cual nunca se abonó ninguna regalía (...) El canon tecnológico se va a pagar por separado 


\section{A modo de breve conclusión}

Creemos que las biotecnologías no constituyen un problema en sí mismo. El problema lo genera la voracidad del mercado -ilimitada en el escenario neoliberal- y las dificultades que tienen actualmente los Estados y el sistema internacional para ponerle límites a los abusos de posición dominante en el mercado y a la imposición de reglas de juego internacionales al servicio de las corporaciones angurrientas que no evalúan los daños que generan, simplemente porque no les importa.

Para finalizar queremos hacerlo con palabras de Joseph Stiglitz, para quien es un sinsentido que quienes negocian los acuerdos comerciales prioricen a los lobbistas por sobre los demás afectados. A su juicio los derechos fundamentales deben ponerse por encima de los intereses empresariales. Para ello "las discusiones sobre criterios de aplicación de los derechos de propiedad intelectual no deben tomarse en el seno de la OMC, sino dentro de una WIPO reformada, esto es, de una Organización Mundial de la Propiedad Intelectual en la que la ciencia cuente tanto como la voz de las empresas, la de los consumidores tanto como la de los productores, la de los países en vías de desarrollo tanto como la de las naciones desarrolladas." 32

Efectivamente, algunos países lograron ponerle límites a los derechos de patentes, ganando votaciones en organizaciones como la OMS. Ese parece el camino que se deberá seguir para aprovechar los beneficios de las biotecnologías, sin tener que padecer las consecuencias dañinas previsibles y evitables.

\section{Bibliografía}

Alexy, Robert El concepto y la validez del derecho, Barcelona, Gedisa, |99|.

Casella, Aldo P. "Argentina: Ley de semillas, propiedad intelectual y políticas públicas", http://wwww.biodiversidadla. org/Portada_Principal/Documentos/Argentina_Ley_de_semillas_propiedad_intelectual_y_politicas_publicas.

Gras, Carla y Hernández, (comps.) El agro como negocio, Buenos Aires, Biblos, 2013.

Flax, Javier Ética, política y mercado. En torno a las ficciones neoliberales, Los Polvorines, UNGS, 20I3, con reedición en 2014 .

Flax, Javier Política científica, interdisciplina y derechos humanos, Buenos Aires, Biblos, 20I4, reeditado en 2015.

de la semilla, porque el gen es de Monsanto, que dará la licencia de uso, mientras que el germoplasma es de cada semillero (...). Cuando se entregue soja Intacta al acopio zonal o al exportador, se deberá avisar que se trata de grano que contiene esa tecnología. El receptor chequeará en el sistema si el productor que envía esos camiones pagó antes el canon tecnológico o no. Y, si no lo hizo, deberá pagarlo en esa instancia. "Ser o no ser, esa es la cuestión", publicado en el suplemento Clarín Rural el 23 de febrero de 2013.

32 Ibídem, pág. 172. 
García, Rolando "Conceptos básicos para el estudio de sistemas complejos", en Enrique LEFF, Los problemas del conocimiento y la perspectiva ambiental del desarrollo, México, Siglo XXI, 1986.

García, Rolando "La investigación interdisciplinaria de sistemas complejos", Centro de Estudios Avanzados - UBA - Serie Materiales I/91, 1991.

García, Rolando Sistemas complejos, Conceptos, métodos y fundamentación epistemológica de la investigación interdisciplinaria, Barcelona, Gedisa, 2006.

O'Donnell, Santiago ArgenLeaks. Los cables de Wikileaks sobre la Argentina, de la A a la Z, Buenos Aires, Sudamericana, 2011 .

Poth, Carla "Reconstruyendo la institucionalidad del modelo biotecnológico", en Carla Gras y Valeria Hernández (comps.) El agro como negocio, Buenos Aires, Biblos, 2013,

Stiglitz, Joseph Cómo hacer que funcione la globalización, Buenos Aires, Taurus, 2006.

Varsavsky, Oscar Ciencia, política y cientificismo, Buenos Aires, CEAL, 1969, reeditado en Varsavsky, Obras escogidas, publicada por Ediciones de la UNLa, Lanús, 2012.

Verbitsky, Horacio "El predador", http://www.pagina I 2.com.ar/diario/elpais/subnotas/I22647-39235-2009-04-05. html

Warren, Michael and Pisarenko, Natacha "Argentines Link Health Problems to Agrochemicals", The Associated Press, Oct. 20, 2013. 